\title{
Free fatty acids-induced neutrophil extracellular traps lead to dendritic cells activation and $T$ cell differentiation in acute lung injury
}

\author{
Wei Chen ${ }^{1,2, *}$, Hong Chen ${ }^{1,2,}{ }^{*}$, Zhi-Tao Yang ${ }^{3}$, En-Qiang Mao ${ }^{3}$, Ying Chen ${ }^{3}$, Er-Zhen Chen ${ }^{3}$ \\ ${ }^{1}$ Department of Pulmonary and Critical Care Medicine, Ruijin Hospital, Shanghai Jiao Tong University School of \\ Medicine, Shanghai 200025, China \\ ${ }^{2}$ Institute of Respiratory Diseases, School of Medicine, Shanghai Jiao Tong University, Shanghai 200025, China \\ ${ }^{3}$ Department of Emergency, Ruijin Hospital, Shanghai Jiao Tong University School of Medicine, Shanghai 200025, \\ China \\ ${ }^{*}$ Co-first authors
}

Correspondence to: Er-Zhen Chen, Ying Chen; email: cez10732@rih.com, https://orcid.org/0000-0001-7835-6080; cy11530@rih.com, https://orcid.org/0000-0002-2688-8736

Keywords: free fatty acids, neutrophil extracellular traps, acute lung injury, dendritic cells, CD4+ T cells

Received: May 4, $2021 \quad$ Accepted: November 22, $2021 \quad$ Published: December 27, 2021

Copyright: (C) 2021 Chen et al. This is an open access article distributed under the terms of the Creative Commons Attribution License (CC BY 3.0), which permits unrestricted use, distribution, and reproduction in any medium, provided the original author and source are credited.

\section{ABSTRACT}

This study aimed to investigate whether free fatty acids (FFAs) could induce the release of neutrophil extracellular traps (NETs), as well as the mechanism of FFAs-induced NETs in acute lung injury (ALI). FFAs were used to induce NETs production. The reactive oxygen species (ROS) production was detected after FFA and NADPH oxidase inhibitor treatments. The association between FFAs-induced NETs and the activation of p38, ERK, and JNK pathways was investigated. The effect of FFAs-induced NETs on the dendritic cells (DCs) activation and $T$ cell differentiation was investigated. FFAs could induce neutrophils to produce NETs. FFAs significantly promoted ROS production and increased the expression of ERK, p38 and JNK, and treatment of the inhibitors of NAPDH oxidase (DPI), p38 (SB202190), ERK1/2 (U0126) and JNK (SP600125) inhibited FAAs-induced NETs production. FFAs induced NETs could promote DCs activation and consequently led to the differentiation of primary CD4+ T cells into Th1 and Th17 cells and the release of IL-1 $\beta$, IL-12 and TNF- $\alpha$. FFAs are capable of inducing NETs via NOX, ERK, p38 and JNK pathways. FFA-induced NETs further lead to DCs activation and T cell differentiation, which can well explain the mechanism of ALI caused by FFAs.

\section{INTRODUCTION}

Acute lung injury (ALI) is a critical illness syndrome characterized by excessive uncontrolled inflammation and apoptosis [1]. As the most severe form of ALI, acute respiratory distress syndrome (ARDS) is an acute and progressive diffuse inflammatory lung injury caused by various intrapulmonary or extrapulmonary factors. The clinical manifestations of ARDS are respiratory distress and refractory hypoxemia [2]. ARDS patients account for $10 \%$ of intensive care unit (ICU) patients, and its mortality rate is up to 35-46\% [3]. Due to lack of accepted diagnostic test, ALI/ARDS remains a challenging entity for clinical investigation. Therefore, elucidation of key mechanism underlying lung injury will have great significance in improving clinical outcomes.

Neutrophils are an important defense against the invasion of pathogenic microorganisms and play a crucial role in the process of non-specific immunity. Accumulating evidence has confirmed that neutrophils are key players in the inflammatory response that characterizes ALI [4]. When neutrophils are activated by pathogenic microorganisms, their structure changes and breaks down into DNA chains with various proteins embedded in the skeleton. This complex three-dimensional network is called neutrophil extracellular traps (NETs) which were first discovered by Brinkmann et al. in 2004 [5]. In recent 
years, NETs have become a research hotspot in immunology and medicine [6-8]. When pathogenic microorganisms invade the body, NETs will transfer to the infected site to capture them, and release high concentrations of histones, antimicrobial peptides and other antimicrobial substances to wrap and kill pathogens [9]. On the other hand, it has been reported that NETs are harmful to cells and tissues, and uncontrolled NETs can lead to ALI, ARDS, and autoimmune diseases [10-12]. A recent study has also shown that excessive accumulation of NETs in inflammatory lung tissue can aggravate the inflammatory response [13].

Free fatty acids (FFAs) are the product of neutral fat metabolism, which are the main cause of lung injury [14]. It has been found that the content of FFAs in serum of mice with lung injury increased significantly [15]. Nevertheless, at present, the mechanism underlying FFAs-induced lung injury is still unclear. In addition, the effect of FFAs on the production of NETs has been reported. For instance, Alarcon et al. found that oleic acid (OA) and linoleic acid (LA) could induce the release of NETs [16], and Khan et al. showed that palmitic acid (PA) and OA could induce the release of NETs in a dose-dependent manner [17]. However, there is no clear evidence that FFAs play a role in ALI by inducing the production of NETs.

Dendritic cells (DCs) are the bridge between innate immune response and adaptive immune response, and take part in the elimination of virus or bacterial infection $[18,19]$. Both lung infection and bleomycin induced lung injury are caused by DCs [20]. Furthermore, the activation of DCs induced by NETs leads to Th1 differentiation, and the compensatory effort of DCs can resist the ongoing inflammation [21, 22]. Whether NETs can induce ALI by further activating DCs and promoting $\mathrm{T}$ cell differentiation remains an open question.

In the present study, neutrophils were isolated from human peripheral blood, and NETs were induced by different concentrations of FFAs. Moreover, the association between FFAs-induced NETs and p38, ERK and JNK pathways was investigated. Furthermore, the effect of NETs induced by FFAs on the DCs activation and $\mathrm{T}$ cell differentiation was explored. Above all, we hypothesized that FFAs could induce the release of NETs and lead to DCs activation and $\mathrm{T}$ cell differentiation, thus playing a key role in ALI.

\section{MATERIALS AND METHODS}

\section{Human neutrophil preparation and detection}

This study was approved by the Ethic Committee of Ruijin Hospital, Shanghai Jiao Tong University. After informed consent, the peripheral blood was collected from healthy volunteers and moderately lysed with red blood cell lysis buffer. The unlysed cells were immediately collected by centrifugation. Human neutrophil enrichment kit (StemCell, USA) was used to separate human primary neutrophils according to the instruction, and the isolated neutrophils were resuspended in Dulbecco's Modified Eagle Medium (DMEM, Hyclone, South Logan, UT) containing with $10 \%$ of fatal bovine serum (FBS, Gibico, Ground Island, NY, USA). The neutrophils suspension was added with trypan blue staining solution (Solarbio) and stained at room temperature for 3-5 min. The stained cells were dropped onto the slide and the survival rate of neutrophils was observed under the microscope. It could meet the requirements of subsequent experiments when the survival rate was above $90 \%$. The concentration of neutrophils was determined by using hemocytometer (Abbott Diagnostics, Santa Clara, CA) and diluted into $2 \times 10^{6}$ cells $/ \mathrm{ml}$ with DMEM medium containing $10 \%$ FBS. The neutrophils were coated on the slide and fixed with methanol for 1-3 min, then stained with Giemsa-Wright staining solution (Solarbio, Beijing, China) and cytox solution (Solarbio) respectively. The morphology of neutrophils was observed under the microscope. Moreover, the neutrophil purity was determined by detecting CD11b+CD16+ using flow cytometry. Briefly, neutrophils were incubated with APC anti-human $\mathrm{CD} 11 \mathrm{~b}+\mathrm{CD} 16+$ antibody for $60 \mathrm{~min}$. Then CD11b+CD16+ cells were detected by BD FACS Calibur flow cytometer (Becton Dickenson, Mississauga, CA, USA).

\section{Induction of NETs by FFAs}

Neutrophils were inoculated into cell plates pretreated with polylysine and cultured in cell incubator for 45 min with a humidified $5 \% \mathrm{CO}_{2}$ atmosphere at $37^{\circ} \mathrm{C}$. After adherent growth, the cells were stimulated with different concentrations $(20,100,200$, and $300 \mu \mathrm{M})$ of $\mathrm{OA}$ and PA for $3 \mathrm{~h}$. The NETs were carefully washed with PBS to remove possible products of neutrophil activation or degranulation and then partially digested by a restriction enzyme AluI (Thermo Fisher Scientific) at $4 \mathrm{U} / \mathrm{ml}$. The digest was performed at $37^{\circ} \mathrm{C}$ for $20 \mathrm{~min}$ and centrifuged at $4^{\circ} \mathrm{C}$ for $10 \mathrm{~min}$ to remove cells and debris. The NETs were collected for subsequent analysis.

\section{Time course of NETs release after stimulation with FFAs}

Freshly isolated neutrophils were seeded in 96-well black plates in the presence of a non-cell-permeant DNA binding dye Sytox Green ( $5 \mu \mathrm{M}$, Invitrogen, Saint Aubin, 
France). After stimulated with increasing concentrations of $\mathrm{OA}$ and $\mathrm{PA}$ at $37^{\circ} \mathrm{C}$ with $5 \% \mathrm{CO}_{2}$ in the dark, DNA release was followed by measuring Sytox green fluorescence at $0,30,60,90,120,150,180,210$, and 240 $\mathrm{min}$ in a microplate fluorescence reader $\left(\operatorname{Tristar}^{\mathrm{TM}} \mathrm{LB} 941\right.$ BERTHOLD, Bad Wildbad, Germany).

\section{Immunofluorescence labeling and observation of NETS}

After treatment with $4 \%$ paraformaldehyde for $20 \mathrm{~min}$, NETs were incubated with anti-myeloperoxidase (MPO) antibody [EPR20257] (ab208670, Abcam, Cambridge, MA), and anti-neutrophil elastase (NE) antibody [EPR7479] (ab131260, Abcam), respectively. When the nucleus was stained, the NETs were observed under the Olympus IX81 inverted fluorescence microscope and the images were collected. Three different visual fields were randomly selected under the same conditions, and the total cells number in a single visual field was calculated by Image $J$ 1.8.0 software (National Institutes of Health, USA). The NETs productivity was calculated according to the ratio of the NETs number in visual field to the total cells number.

\section{Quantitative analysis of NETs-DNA content}

The quantitative analysis of NETs-DNA was performed by fluorescence quantitative method according to the Quant-iT PicoGreen dsDNA Assay Kit (Invitrogen, USA) instructions. Then, the content of NETs-DNA was detected by fluorescence microplate reader (Thermo, USA).

\section{Detection of NADPH oxidase-mediated reactive oxygen species (ROS) production}

The 2,7-Dichlorofluorescein diacetate (DCFH-DA, Sigma, USA) was used as fluorescent probe to detect ROS. Neutrophils was inoculated into 96-well plates and treated with DCFH-DA for $30 \mathrm{~min}$. After pretreated with DPI, a NADPH oxidase inhibitor, the neutrophils were incubated with different concentrations of $\mathrm{OA}$ for $3 \mathrm{~h}$ at $37^{\circ} \mathrm{C}$. Finally, fluorescence microplate reader (Thermo) was used to detect the content ROS at excitation and emission wavelengths of $488 \mathrm{~nm}$ and $525 \mathrm{~nm}$.

\section{Detection of lactate dehydrogenase (LDH) release}

LDH release assay kit (Beyotime, China) was used for cytotoxicity test, and the operation was carried out according to the instructions. The optical density of 96well plate was measured at $490 \mathrm{~nm}$ wavelength by a microplate reader (Biotek, USA), the content of LDH in culture supernatant was determined, and the cell death rate was calculated.

\section{Detection of cell apoptosis by flow cytometry}

Cell apoptosis was detected using the Annexin VFITC/PI apoptosis detection kit (Yeasen, Pudong, Shanghai, China). Briefly, after exposing cells to different concentrations of OA for $24 \mathrm{~h}$, cells were harvested and washed with PBS buffer. Then, cells were resuspended in $1 \times$ binding buffer and incubated with Annexin V-FITC/PI buffer (containing $5 \mu \mathrm{L}$ Annexin $\mathrm{V}$-FITC and $10 \mu \mathrm{L}$ propidium iodide (PI)) in the dark at room temperature for $15 \mathrm{~min}$. Cell apoptosis was detected by flow cytometry using BD FACS Calibur (Becton Dickenson), and the data were analyzed using CellQuest Pro software (Becton Dickenson).

\section{Western blot analysis}

Western blot was used to detect the effect of OA on p38, ERK, and JNK signaling pathways. Lysate buffer was added in the ratio of $200 \mu \mathrm{L} 2 \times$ SDS loading buffer to $1 \times 10^{7}$ cells, and vortex oscillates until the cell mass was not visible. The homogenate was collected after centrifugation, and the protein concentration of supernatant was measured by Bradford method. Equal amounts of proteins $(40 \mu \mathrm{g})$ was loaded on $10 \%$ SDS-PAGE and then transferred to nitrocellulose membranes (Millipore, Billerica, MA, USA). After blocked with 5\% nonfat milk, the membranes were incubated with primary antibodies against JNK, p-JNK, ERK, p-ERK, p38, p-p38, and GAPDH (1:1000 dilution; Solarbio) overnight at $4{ }^{\circ} \mathrm{C}$. Then the membranes were incubated for $1 \mathrm{~h}$ with horseradish peroxidase-conjugated secondary antibodies (1:3000 dilution; Solarbio). Protein bands were visualized using an ECL detection kit (Pierce, Thermo Scientific, Waltham, USA). Proteins were quantified by densitometry with the Image J 1.8.0 software (National Institutes of Health, USA).

\section{Treatment of inhibitors}

In order to verify the mechanism of NETs production, neutrophils were pretreated for $30 \mathrm{~min}$ with the following inhibitors: NADPH oxidase inhibitor (DPI), NETs inhibitor (DNase I), p38-specific inhibitor (SB202190), ERK1/2-specific inhibitor (U0126), and JNK-specific inhibitor (SP600125). Then neutrophils were incubated with $\mathrm{OA}$ for $3 \mathrm{~h}$ at $37^{\circ} \mathrm{C}$. Finally, the NETs content was determined by fluorescence quantitative method.

\section{Culture and detection of DCs}

Peripheral blood mononuclear cells were isolated from healthy volunteers and resuspended in RPMI 1460 medium (Gibco) containing 10\% FBS. Mononuclear 
cells $\left(3 \times 10^{6} / \mathrm{mL}\right)$ were seeded on 6 -well plates with 2 $\mathrm{mL} / \mathrm{well}$, and then cultured in the incubator for $4 \mathrm{~h}$. After the cells adhered to the wall, the full amount of culture medium was replaced with RPMI-1640 medium containing granulocyte-macrophage colony-stimulating factor (GM-CSF) and IL-4. Half of the old medium was replaced every other day, and DCs were collected on the 6th day. DCs were then detected by flow cytometry. In brief, DCs were incubated with APC anti-human CD11c antibody for $60 \mathrm{~min}$. Then CD11c+ cells were detected by BD FACS Calibur flow cytometer (Becton Dickenson), and more than $70 \%$ purity could be used for subsequent experiments.

\section{The influence of NETs on DCs}

NETs were prepared by stimulating with freshly isolated neutrophils with OA. DCs $\left(1 \times 10^{6} / \mathrm{mL}\right)$ were seeded on 6-well plates and stimulated with or without freshly prepared NETs for $24 \mathrm{~h}$. DCs were incubated with CD40, CD86 and HLA-DR antibodies and resuspended with $2 \%$ paraformaldehyde, then detected by FACSCalibur flow cytometer (Becton Dickenson) within $24 \mathrm{~h}$. The expression of cytokines (IL-1 $\beta$, IL-12, and TNF- $\alpha$ ) in DCs supernatant were tested by commercial enzyme-linked immunosorbent assay kits (ELISA kits, Assay Design, Ann Arbor, MI) according to the instructions of manufacturers. In addition, to avoid direct effects of OA on DCs, the influence of OA on DCs apoptosis and DCs-DNA content was detected by flow cytometry and quantitative analysis of DCsDNA.

\section{The influence of DCs on $\mathrm{CD4}^{+} \mathrm{T}$ cells}

$\mathrm{CD}^{+} \mathrm{T}$ cells were isolated from human peripheral blood by immunomagnetic beads technique. DCs $(4 \times$ $\left.10^{4}\right)$ stimulated with or without NETs were collected and co-cultured with primary $\mathrm{CD} 4^{+} \mathrm{T}$ cells $\left(2 \times 10^{5}\right)$ in 24 well-plates at the ratio of 1:5. The primary $\mathrm{CD}^{+} \mathrm{T}$ cells were set as control. On the first day of co-culture, anti-human CD28 antibody $(2 \mu \mathrm{g} / \mathrm{mL})$ and IL-2 cytokines $(10 \mathrm{ng} / \mathrm{mL})$ were added to each well, and on the third day, half of the medium was changed. For activation of $\mathrm{CD}^{+} \mathrm{T}$ cells, on the fourth day of coculture, Phorbol 12-myristate 13-acetate (PMA, 50 $\mathrm{ng} / \mathrm{mL}$, Sigma), ionomycin (1 $\mu \mathrm{g} / \mathrm{mL}$, Sigma) and brefeldin A (BFA, $10 \mu \mathrm{g} / \mathrm{mL}$, Sigma) were added to the culture plate and incubated at $37^{\circ} \mathrm{C}$ for $5 \mathrm{~h}$. The proportion of Th1 and Th17 was detected by flow cytometry.

\section{Statistical analysis}

SPSS 20.0 (SPSS, Inc., Chicago, IL) and GraphPad Prism 7 (GraphPad Software Inc., San Diego, CA,
USA) were used for statistical data analysis. The differences between the two groups were compared using the $T$-test, while ANOVA was used for multigroup comparison. $P<0.05$ indicates the significant results.

\section{RESULTS}

\section{Identification of neutrophils}

To observe the morphology of neutrophils, GiemsaWright and cytox fluorescent nuclear staining were used. The structure of neutrophils could be clearly seen under the microscope, and the nucleus was divided into 2-5 lobes (Figure 1A, 1B). Moreover, the results of flow cytometry showed that purity of neutrophils was more than $80 \%$ (Figure 1C).

\section{FFAs induced the formation of NETs}

In order to confirm that FFAs could induce the formation of NETs, we incubated neutrophils with different concentrations of OA and PA. As shown in Figure 2A, all concentrations of FFAs could induce neutrophils to produce NETs, and high dose showed more obvious induction effect. Compared with the control group, the NET-DNA increased significantly after FFAs stimulation (all $P<0.05$ ), and the effects of different concentrations FFAs on NETs formation rate was dose-dependent (Figure 2B). Confocal images of NETs were shown in Figure 2C. Under the microscope, DNA (blue), MPO (green) and NE (red) were obvious co-localized. It could be seen that NETs was a fibrous network structure with DNA as the skeleton and embedded with NE and MPO particles.

\section{NADPH oxidase inhibitor inhibited OA-induced ROS production}

The formation of NETs was closely related to the ROS, which was the product activated by NADPH oxidase. When neutrophils were pretreated with DPI, a NADPH oxidase inhibitor, for $30 \mathrm{~min}$, the increase of ROS stimulated by $\mathrm{OA}$ decreased significantly $(P<0.05)$ (Figure 3). The results showed that NADPH oxidase might be involved in OA-induced ROS production.

\section{OA treatment increased cell damage}

Extracellular LDH release usually indicated damaged cells. As shown in Figure 4, compared with the control group, OA treatment significantly increased $\mathrm{LDH}$ release in a dose-dependent manner (all $P<0.05$ ), suggesting that the cell activity decreased obviously. Moreover, cell apoptosis was detected after exposing cells to different concentration of OA. The results 
showed that OA treatment significantly prompted cell apoptosis in a dose-dependent manner (all $P<0.05$ ). Collectively, it could be conducted that OA treatment increased cell damage.

\section{OA induced activation of p38, ERK, and JNK pathways}

To investigate the possible mechanism of FFAs-induced lung injury, the effects of OA treatment on the activation of $\mathrm{p} 38$, ERK, and JNK pathways were investigated. As shown in Figure 5, compared with the control group, OA treatment resulted in the significantly increased expression of p-ERK, p-Ep38 and p-EJNK (all $P<0.05$ ), indicating that $\mathrm{OA}$ induced activation of p38, ERK, and JNK pathways.

\section{Inhibitors affect OA-induced NETs}

To further confirm that OA-induced the formation of NETs depended on NADPH oxidase, p38, ERK, and JNK pathways, neutrophils were treated with their corresponding inhibitors. The results showed that pretreatment with NADPH oxidase inhibitor (DPI), ERK1/2 inhibitor (U0126), p38 inhibitor (SB202190) and JNK inhibitor (SP600125) could significantly inhibited the increase of NETs induced by OA (all $P<$ 0.05 , Figure $6 \mathrm{~A}$ ), suggesting that NADPH oxidase as well as p38, ERK, and JNK pathways might be involved in the formation of NETs. Moreover, we found that DNase I (a NET inhibitor) treatment also significantly decreased OA-induced formation of NETs $(P<0.001$, Figure 6B).

\section{OA-induced NETs promote the activation of DCs}

As shown in Supplementary Figure 1, OA treatment had no significant effects on DCs apoptosis and DCsDNA content, indicating that there was direct effect of OA on DCs. To determine whether DCs were activated by NETs, we evaluated the positive expression of CD40, CD86 and HLA-DR on DCs with or without stimulation by NETs for $15 \mathrm{~h}$. As shown in the Figure 7A, compared with DCs without NETs stimulation, stimulated DCs exhibited higher levels of CD40, CD86 and HLA-DR (all $P<0.05$ ). The results indicated that OA-induced NETs could promote the maturation and activation of DCs. In addition, the soluble IL- $1 \beta$, IL- 12 and TNF- $\alpha$ levels in the supernatant of NETs-stimulated DCs were significantly higher than that of DCs without stimulation (all $P<0.05$ ) (Figure 7B).
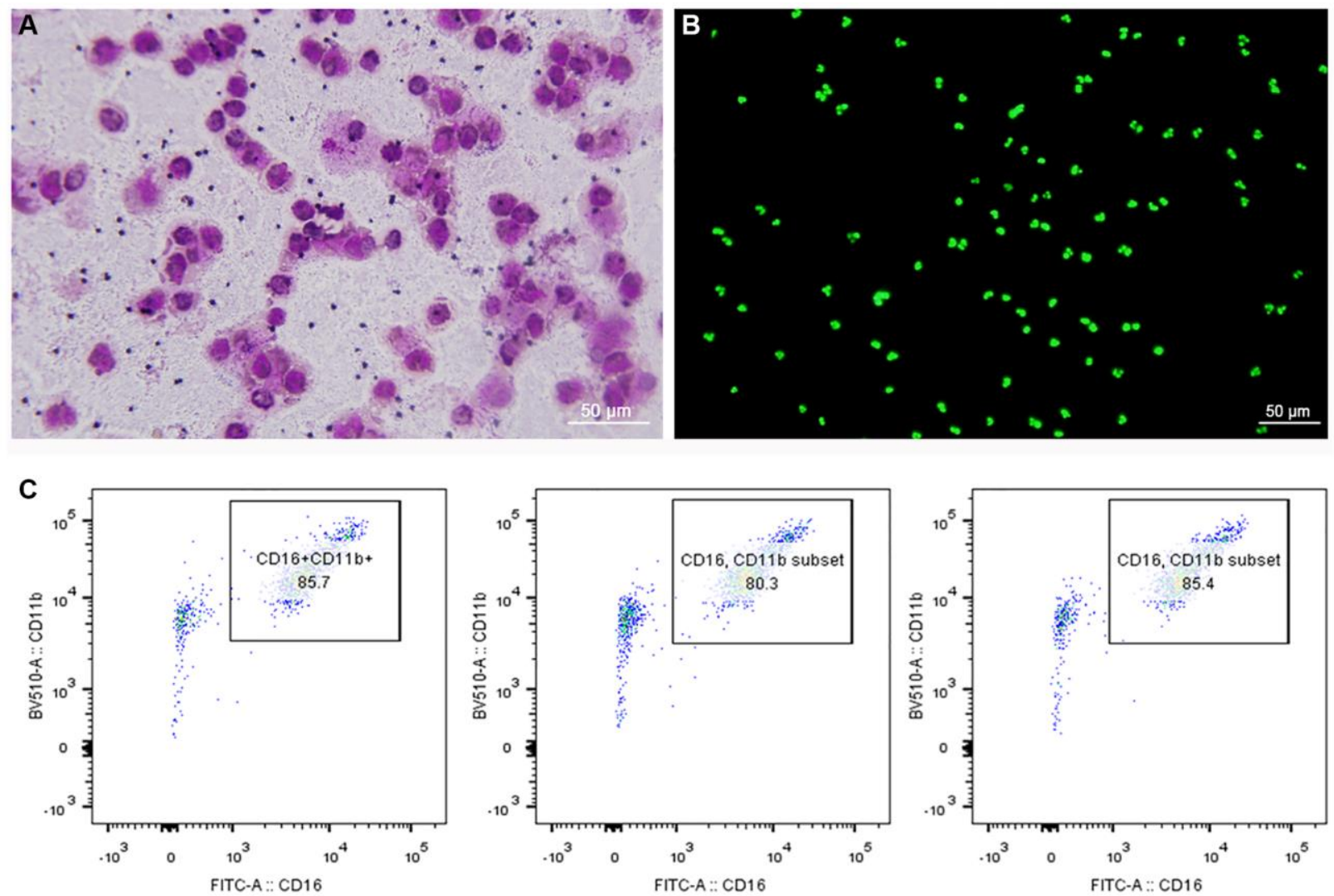

Figure 1. Morphology and purity of neutrophils. (A) Giemsa-Wright staining method. (B) Cytox fluorescent nuclear staining method. (C) Flow cytometry showed that purity of neutrophils was more than $80 \%$. 
It is reported that DCs regulated the differentiation of lymphocytes in a pathogen-specific manner [23-25]. In order to explore the role of DCs in the immune response of Th1 and Th17 cells to NETs, we detected the DCs with or without stimulation by NETs by flow cytometry. As revealed in Figure 7C, there were a few differentiated Th1 and Th17 cells in the only primary
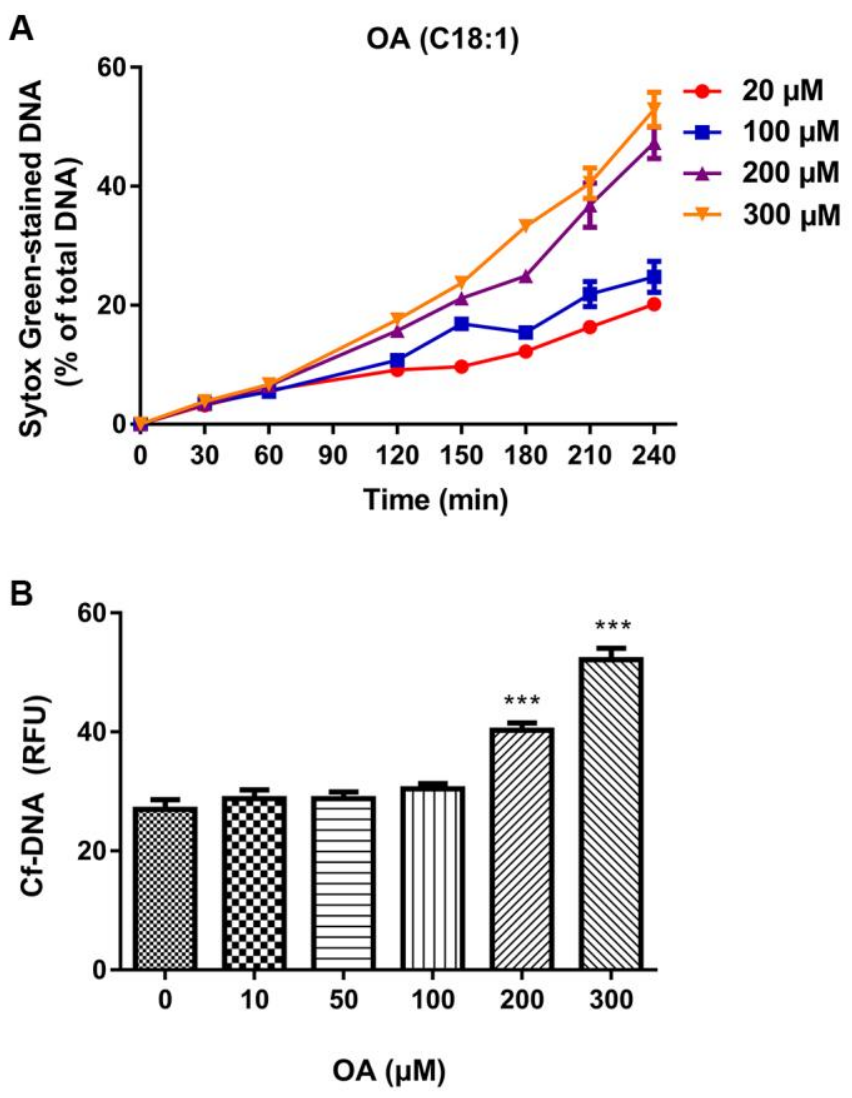

C
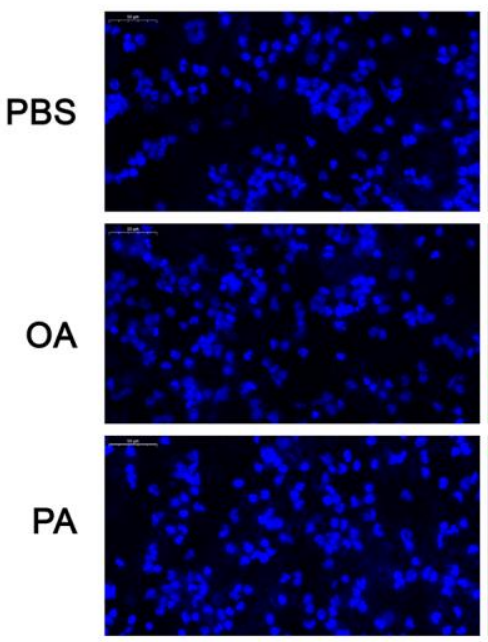
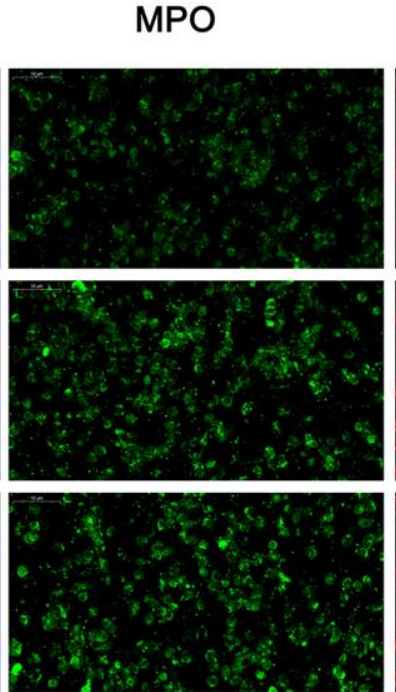

$\mathrm{CD}^{+} \mathrm{T}$ well without DCs. The proportion of Th1 and Th17 cells in the co-culture system of DCs stimulated by NETs and primary $\mathrm{CD}^{+} \mathrm{T}$ was significantly higher than of DCs without stimulated by NETs and primary $\mathrm{CD}^{+} \mathrm{T}(P<0.05)$. These data suggested that NETsstimulated DCs promoted the differentiation of primary CD4 ${ }^{+} \mathrm{T}$ cells into Th1 and Th17 cells.
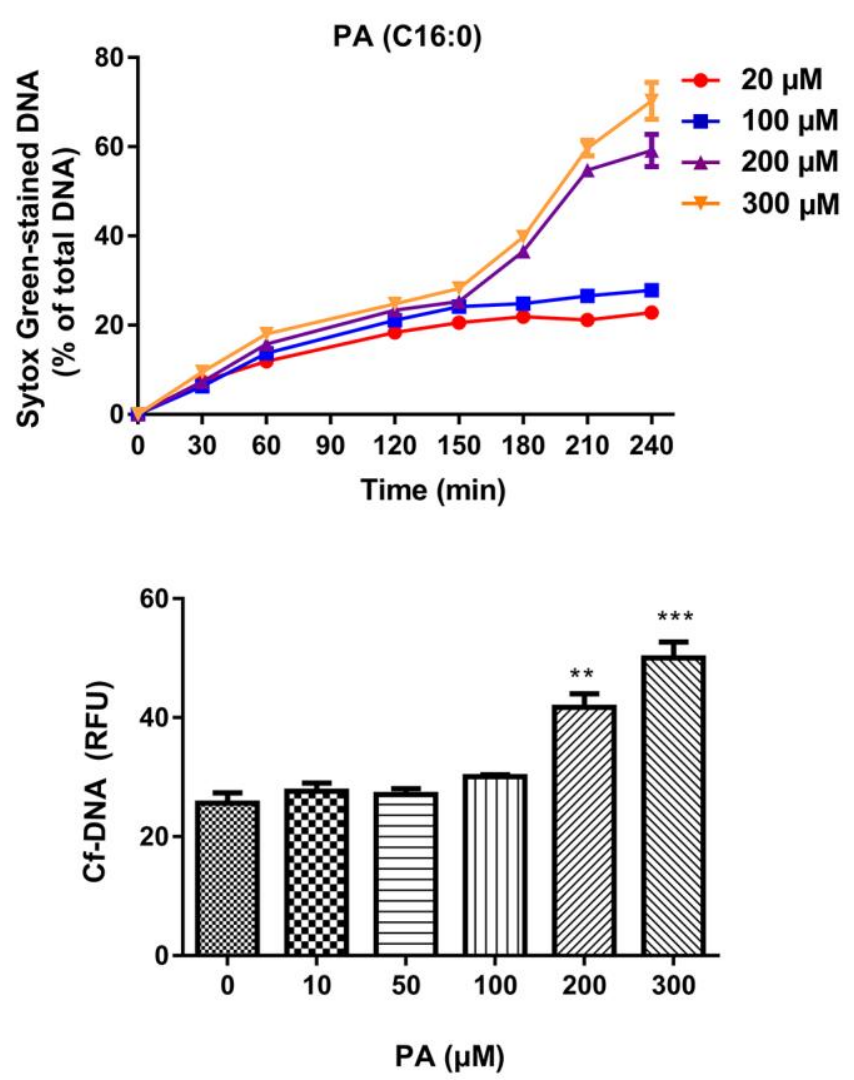

NE
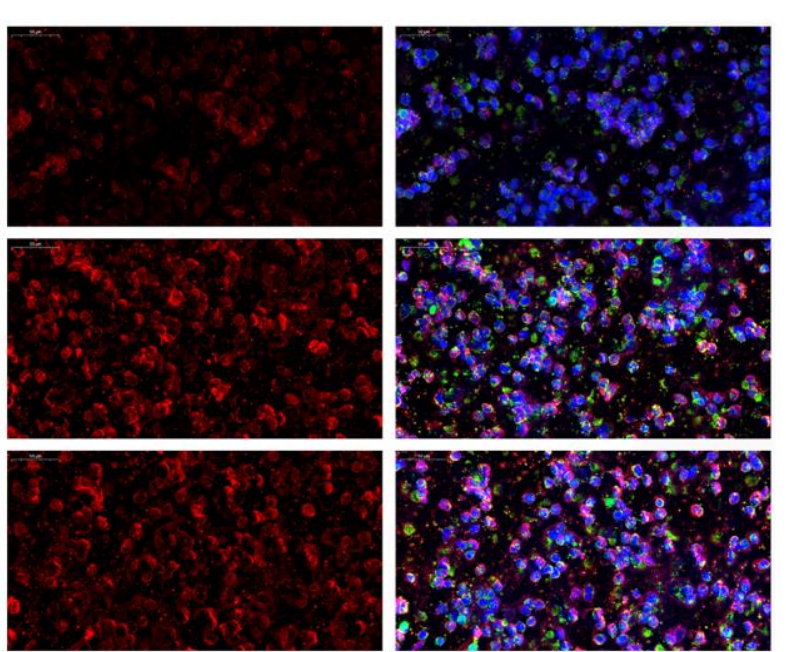

Figure 2. Effects of FFAs on NETs formation. (A) The time curve of NETs formation induced by different concentrations of FFAs. (B) NETs formation rate stimulated by FFAs for $3 \mathrm{~h}$. (C) Confocal images of NETs stimulated by FFAs for 3 h. Abbreviations: FFAs: free fatty acids; NETs: neutrophil extracellular traps. ${ }^{* *} P<0.01$ and ${ }^{* * *} P<0.001$. 


\section{DISCUSSION}

The present study evaluated the mechanism of FFAsinduced NETs in ALI in vitro. We found that FFAs could induce neutrophils to produce NETs. Moreover, FFAs significantly promoted the production of ROS and increased the expression of p-ERK, p-p38 and p-JNK, and treatment of the corresponding inhibitors of NAPDH oxidase, p38, ERK and JNK pathways inhibited FAAs-induced NETs production. Furthermore, FFAs-induced NETs could lead to DCs activation and DCs activated by NETs promoted the differentiation of primary $\mathrm{CD}^{+} \mathrm{T}$ cells to Th1 and Th17 cells. These data suggested the potential role and regulatory mechanism of FFAs-induced NETs in ALI.
NETs have recently become a hot topic for research due to their central role in the development of autoimmune diseases [26]. The formation of NETs is related to the activation of NADPH oxidase complex and the production of ROS, and excessive ROS can cause cell oxidative damage [27]. Consistent with previous findings that PA and OA are capable of inducing NETs through a NOX-dependent pathway [17], our results showed that the enhanced level of ROS stimulated by FAAs decreased significantly after treatment with a NADPH oxidase inhibitor, confirming that FFAs induced NETs formation via NOX-dependent pathway. Moreover, some studies have shown that ROS can promote the production of NETs by activating p38, ERK, and JNK $[17,28]$. The activation of p38MAPK,

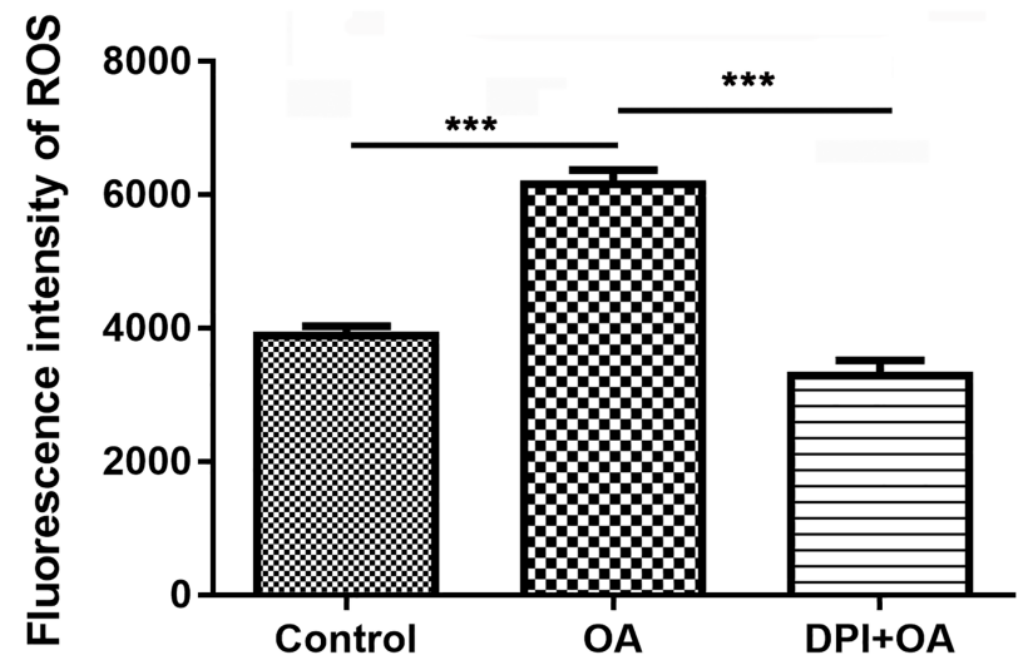

Figure 3. DPI inhibited OA-induced ROS production. Abbreviations: DPI: NAPDH oxidase inhibitor; OA: oleic acid; ROS: reactive oxygen species. ${ }^{* * *} P<0.001$.
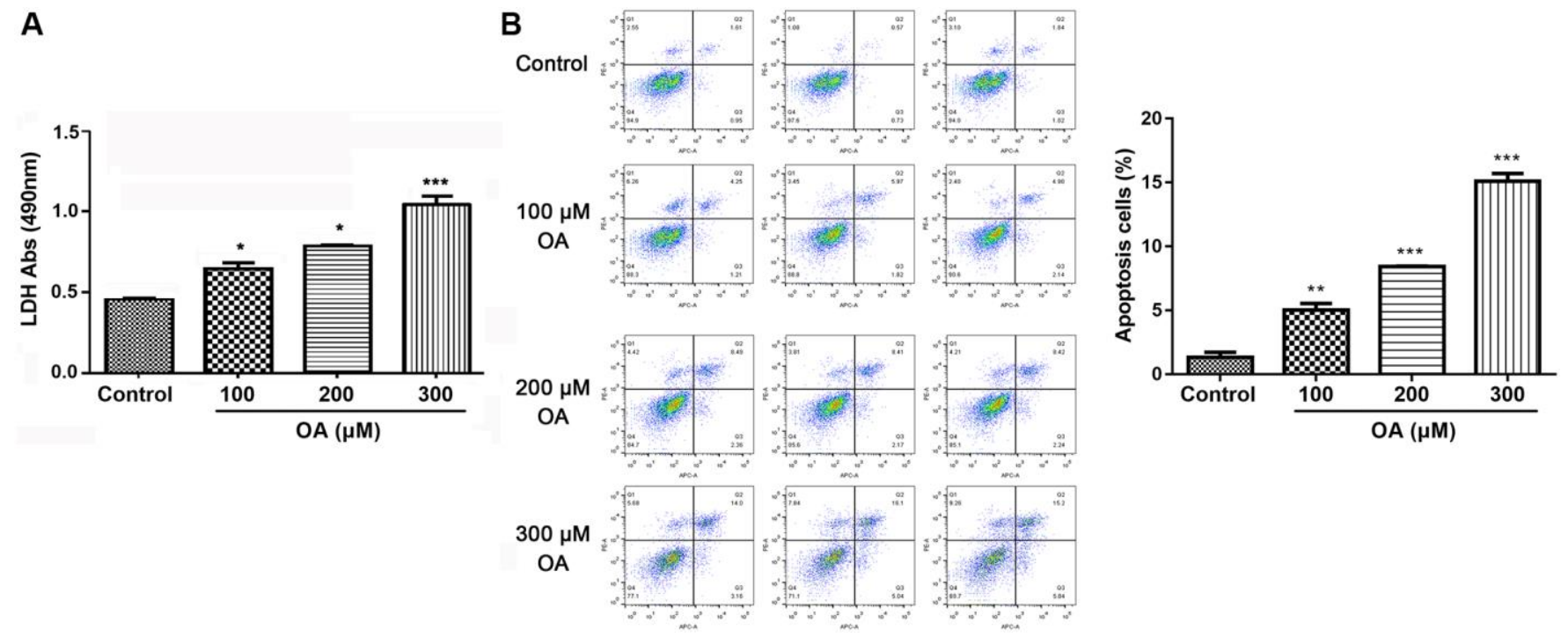

Figure 4. OA treatment increased cell damage. (A) Effect of OA on LDH release. (B) Effect of OA on cell apoptosis. Abbreviations: OA: oleic acid; LDH: lactate dehydrogenase. ${ }^{*} P<0.05,{ }^{* *} P<0.01$, and ${ }^{* * *} P<0.001$. 
JNK, and ERK are closely related to gene expression, cell proliferation and death [29]. It has been reported that colchicine treatment can protect against LPSinduced lung damage in rats via regulating the activation of p38, ERK, and JNK [30]. We found that the p38, JNK and ERK pathways were obviously activated during the formation of NETs induced by FFAs. It can be speculated that FAAs may induce NETs in ALI through regulating the activation of p38, ERK, and JNK pathways.

Furthermore, it is found that NETs can induce T cells differentiation, and this process does not arise from the direct effect of NETs on T cells, but is mediated by DCs [22]. DCs are implicated in the regulating the

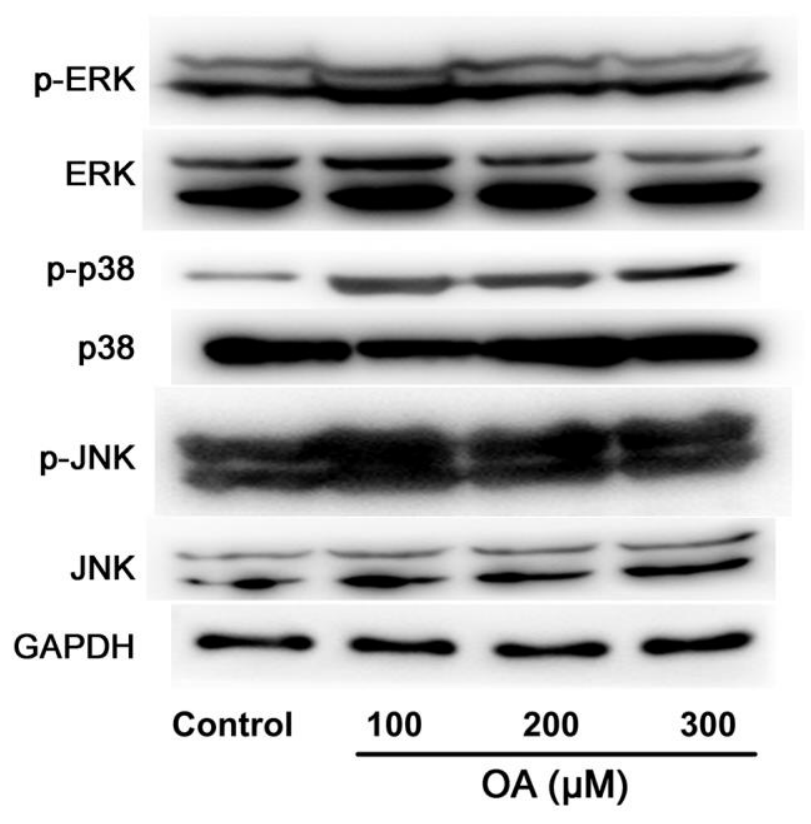

p-ERK/ERK

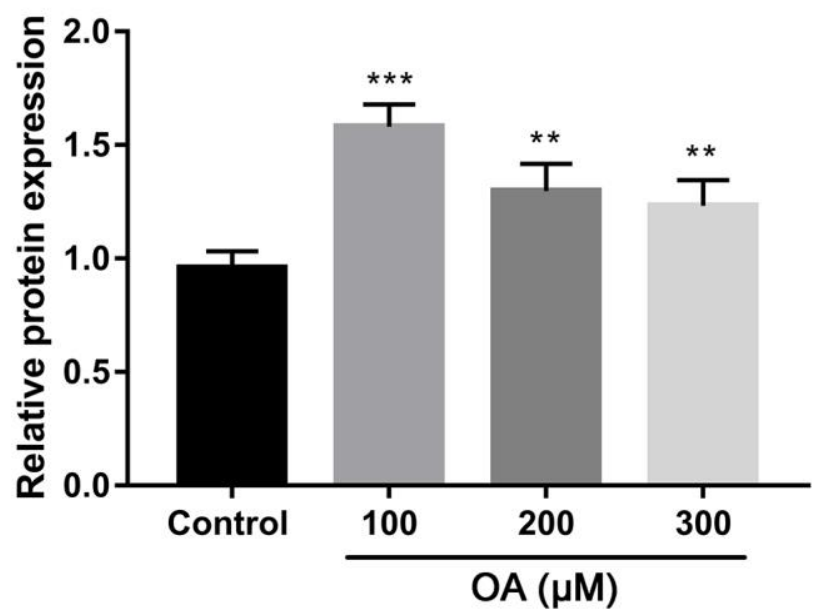

inflammatory and regulatory responses to fungal infection [23, 25]. DCs have weak ability to capture and process antigens in immature state. When stimulated by external environment, DCs can activate primary $\mathrm{T}$ cells and start adaptive immune response, which plays an important role in respiratory diseases [31]. In addition, DCs are the component of innate immune response and the most powerful antigen presenting cells, which are the key link between innate immunity and adaptive immunity. DCs stimulated by NETs produced more IL-1 $\beta$, IL-12 and TNF- $\alpha$, indicating that DCs were activated. At present, many studies have confirmed the role of activated DCs in lung injury [32, 33]. In addition, our results showed that DCs activated by NETs promoted the differentiation of primary CD4+

\section{p-ERK/ERK}

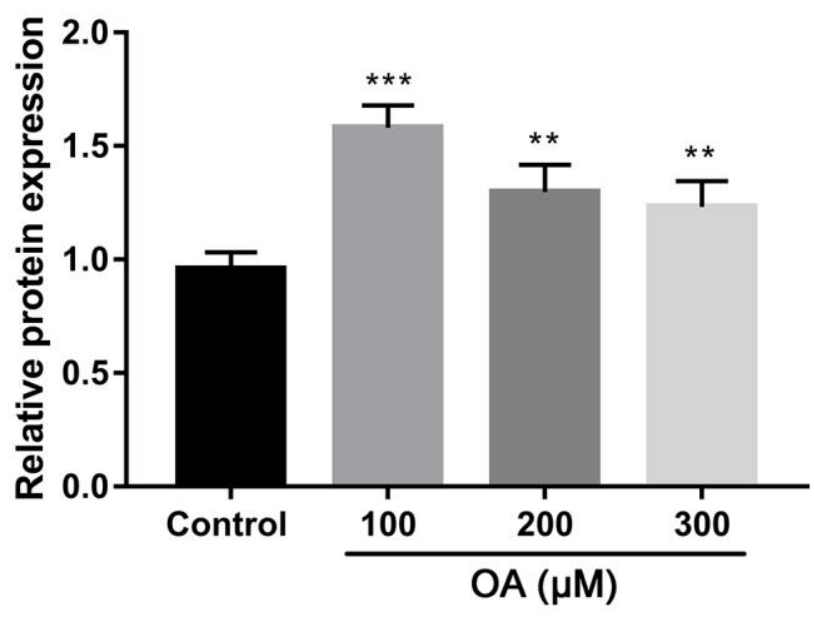

p-JNK/JNK

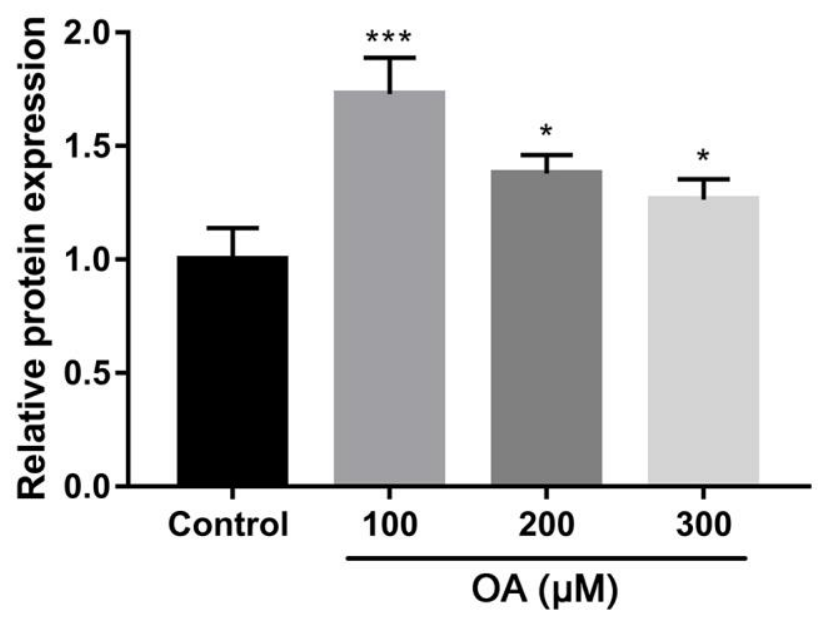

Figure 5. Effect of OA on p38, ERK and JNK signaling pathways. Abbreviation: OA: oleic acid. ${ }^{*} P<0.05,{ }^{* *} P<0.01$, and ${ }^{* * *} P<0.001$. 
A

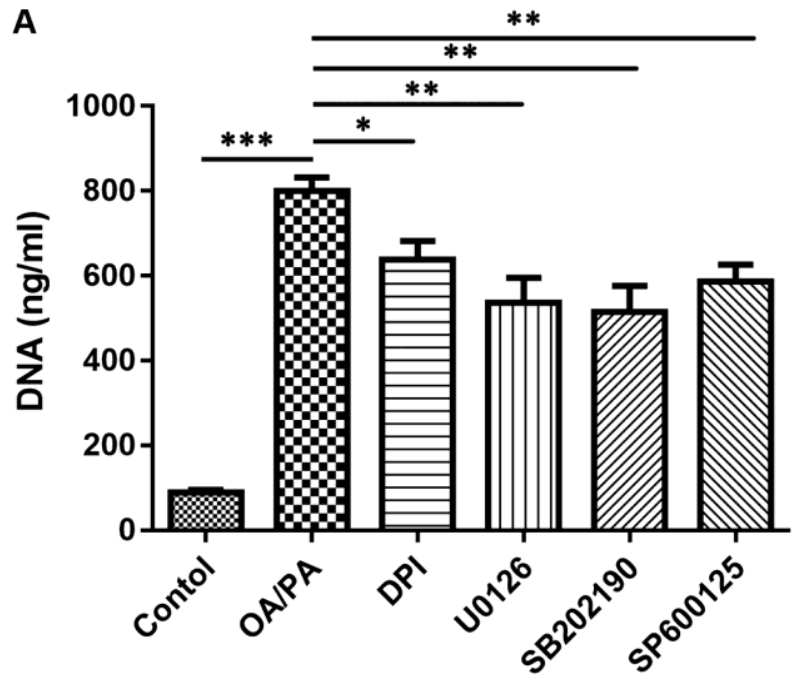

B

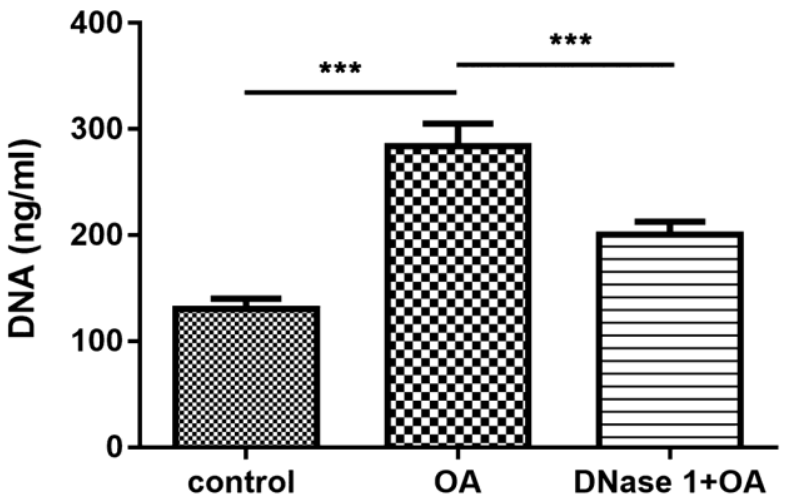

Figure 6. Inhibitors reduced the formation of NETs induced by OA. (A) Effect of DPI, U0126, SB202190, and SP600125 on OA-induced formation of NETs. (B) Effect of DNase I on OA-induced formation of NETs. Abbreviations: OA: oleic acid; NETs: neutrophil extracellular traps; DPI: NADPH oxidase inhibitor; DNase I: NETs inhibitor; SB202190: p38-specific inhibitor; U0126: ERK1/2-specific inhibitor; SP600125: JNK-specific inhibitor. ${ }^{*} P<0.05,{ }^{* *} P<0.01$, and ${ }^{* * *} P<0.001$, respectively.
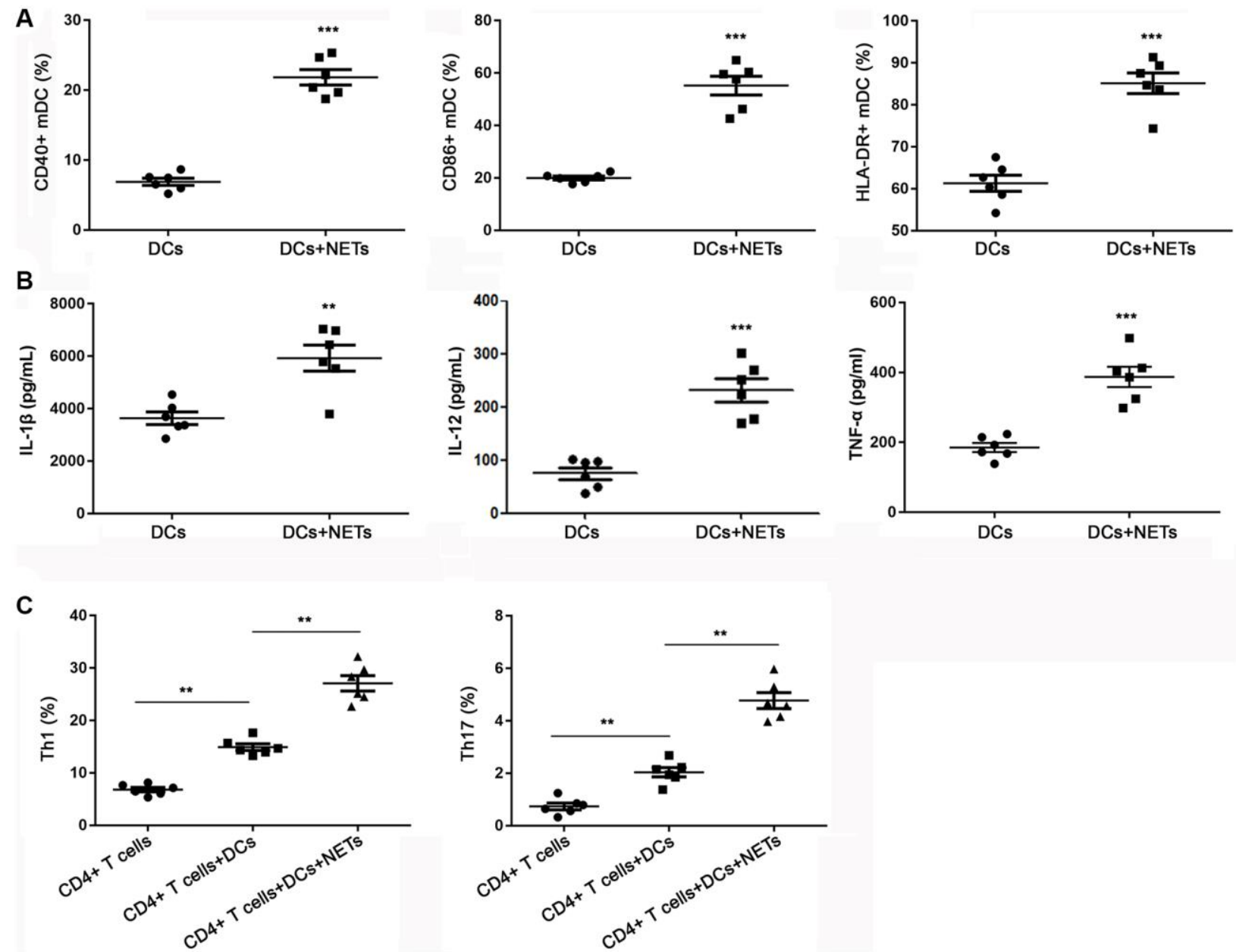

Figure 7. FFAs induced NETs promote the activation of DCs. (A) DCs stimulated with NETs could increase the expression of CD40, CD86 and HLA-DR. (B) DCs stimulated with NETs could improve the release level of IL-1B, IL-12 and TNF- $\alpha$. (C) DCs stimulated with NETs could promote the differentiation of primary $\mathrm{CD}^{+} \mathrm{T}$ into Th1 and Th17 cells. Abbreviations: FFAs: free fatty acids; NETs: neutrophil extracellular traps; DCs: dendritic cells. ${ }^{* *} P<0.01$ and ${ }^{* * *} P<0.001$. 
T cells to Th1 and Th17 cells. Aberrant Th1 and Th17 cells could activate innate immune cells by producing proinflammatory cytokines, such as IFN $\gamma$, IL-17, and TNF $\alpha$, and metformin is shown to inhibit in the production of cytokines of Th1 and Th17 cells, alleviating the autoimmune diseases including various ALIs [34, 35]. Therefore, we speculate that the activation of DCs and subsequent $T$ cell differentiation may be responsible for FFAs-induced ALI.

Our study certificated the important role and possible of FAAs-induced NETs in the development of ALI and elucidated the correlation between immunity in ALI. However, the correlation between FFAs and ALI as well as the mechanism of FFAs-induced NETs formation in the development of ALI was not confirmed by clinical and in vivo studies. More research is still required to confirm our results.

In conclusion, our findings reveal that FFAs can induce the release of NETs via NOX-dependent pathway as well as p38, ERK and JNK pathways. FFA-induced NETs could further lead to DCs activation and CD4 ${ }^{+}$ $\mathrm{T}$ cell differentiation into Th1 and Th17 cells, thus playing a key role in ALI. The results of this study can well explain the mechanism of lung injury caused by FFAs and provide a new perspective for the clinical research.

\section{AUTHOR CONTRIBUTIONS}

Conceptualization: Wei Chen and Hong Chen; Data curation: Wei Chen and Hong Chen; Formal analysis: Wei Chen, Hong Chen, Zhi-Tao Yang; Investigation: Wei Chen, Hong Chen, En-Qiang Mao; Supervision: Ying Chen and Er-Zhen Chen; Writing original draft: Wei Chen and Hong Chen; Writing review and editing: Ying Chen and Er-Zhen Chen.

\section{CONFLICTS OF INTEREST}

The authors declare no conflicts of interest related to this study.

\section{FUNDING}

This study was funded by the National Natural Science Foundation of China (No. 81772107), Clinical Research Plan of SHDC (No. SHDC2020CR1028B), Shanghai Jiao Tong University School of Medicine (No. DLY201803), Shanghai Science and Technology Innovation Fund (No. 18411950900), and supported by Program for Outstanding Medical Academic Leader to EZC.

\section{REFERENCES}

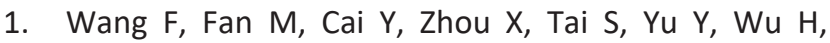
Zhang $Y$, Liu J, Huang S, He N, Hu Z, Jin X. Circular RNA circRIMS1 Acts as a Sponge of miR-433-3p to Promote Bladder Cancer Progression by Regulating CCAR1 Expression. Mol Ther Nucleic Acids. 2020; 22:815-31. https://doi.org/10.1016/j.omtn.2020.10.003 PMID:33230478

2. Fan E, Brodie D, Slutsky AS. Acute Respiratory Distress Syndrome: Advances in Diagnosis and Treatment. JAMA. 2018; 319:698-710. https://doi.org/10.1001/jama.2017.21907 PMID:29466596

3. Matthay MA, Zemans RL, Zimmerman GA, Arabi YM, Beitler JR, Mercat A, Herridge M, Randolph AG, Calfee CS. Acute respiratory distress syndrome. Nat Rev Dis Primers. 2019; 5:18.

https://doi.org/10.1038/s41572-019-0069-0 PMID:30872586

4. Abraham E. Neutrophils and acute lung injury. Crit Care Med. 2003; 31:S195-9. https://doi.org/10.1097/01.CCM.0000057843.47705.E8 PMID: 12682440

5. Brinkmann V, Reichard U, Goosmann C, Fauler B, Uhlemann Y, Weiss DS, Weinrauch Y, Zychlinsky A. Neutrophil extracellular traps kill bacteria. Science. 2004; 303:1532-5. https://doi.org/10.1126/science.1092385 PMID: 15001782

6. Lee $\mathrm{KH}$, Kronbichler A, Park DD, Park Y, Moon H, Kim H, Choi JH, Choi Y, Shim S, Lyu IS, Yun BH, Han Y, Lee $D$, et al. Neutrophil extracellular traps (NETs) in autoimmune diseases: A comprehensive review. Autoimmun Rev. 2017; 16:1160-73.

https://doi.org/10.1016/j.autrev.2017.09.012 PMID:28899799

7. Euler $\mathrm{M}$, Hoffmann $\mathrm{MH}$. The double-edged role of neutrophil extracellular traps in inflammation. Biochem Soc Trans. 2019; 47:1921-30. https://doi.org/10.1042/BST20190629 PMID: 31754705

8. Döring $Y$, Libby $P$, Soehnlein $O$. Neutrophil Extracellular Traps Participate in Cardiovascular Diseases: Recent Experimental and Clinical Insights. Circ Res. 2020; 126:1228-41. https://doi.org/10.1161/CIRCRESAHA.120.315931 PMID:32324499

9. Hakkim A, Fuchs TA, Martinez NE, Hess S, Prinz $H$, Zychlinsky A, Waldmann H. Activation of the Raf-MEKERK pathway is required for neutrophil extracellular trap formation. Nat Chem Biol. 2011; 7:75-7. 
https://doi.org/10.1038/nchembio.496 PMID:21170021

10. Narasaraju T, Yang E, Samy RP, Ng HH, Poh WP, Liew AA, Phoon MC, van Rooijen N, Chow VT. Excessive neutrophils and neutrophil extracellular traps contribute to acute lung injury of influenza pneumonitis. Am J Pathol. 2011; 179:199-210. https://doi.org/10.1016/i.ajpath.2011.03.013 PMID:21703402

11. Marcos V, Zhou-Suckow Z, Önder Yildirim A, Bohla A, Hector A, Vitkov L, Krautgartner WD, Stoiber W, Griese M, Eickelberg O, Mall MA, Hartl D. Free DNA in cystic fibrosis airway fluids correlates with airflow obstruction. Mediators Inflamm. 2015; 2015:408935.

https://doi.org/10.1155/2015/408935

PMID: 25918476

12. Yaqinuddin A, Kashir J. Novel therapeutic targets for SARS-CoV-2-induced acute lung injury: Targeting a potential IL-1 $\beta /$ neutrophil extracellular traps feedback loop. Med Hypotheses. 2020; 143:109906. https://doi.org/10.1016/i.mehy.2020.109906 PMID: $\underline{32505910}$

13. Twaddell SH, Baines KJ, Grainge C, Gibson PG. The Emerging Role of Neutrophil Extracellular Traps in Respiratory Disease. Chest. 2019; 156:774-82. https://doi.org/10.1016/j.chest.2019.06.012 PMID:31265835

14. Chondrogiannis KD, Siontis GC, Koulouras VP, Lekka $\mathrm{ME}$, Nakos G. Acute lung injury probably associated with infusion of propofol emulsion. Anaesthesia. 2007; 62:835-7.

https://doi.org/10.1111/i.1365-2044.2007.05141.x PMID:17635435

15. Kokuho N, Terasaki $Y$, Kunugi $S$, Saito $Y$, Urushiyama H, Terasaki M, Hayashi H, Gemma A, Shimizu A. Analyses of alveolar epithelial injury via lipid-related stress in mammalian target of rapamycin inhibitor-induced lung disease. Lab Invest. 2019; 99:853-65.

https://doi.org/10.1038/s41374-018-0158-9 PMID:30728465

16. Alarcon $\mathrm{P}$, Manosalva $\mathrm{C}$, Carretta MD, Hidalgo $\mathrm{Al}$, Figueroa CD, Taubert A, Hermosilla C, Hidalgo MA, Burgos RA. Fatty and hydroxycarboxylic acid receptors: The missing link of immune response and metabolism in cattle. Vet Immunol Immunopathol. 2018; 201:77-87. https://doi.org/10.1016/i.vetimm.2018.05.009 PMID:29914687

17. Khan MA, Pace-Asciak C, Al-Hassan JM, Afzal M, Liu YF, Oommen S, Paul BM, Nair D, Palaniyar N.
Furanoid F-Acid F6 Uniquely Induces NETosis Compared to C16 and C18 Fatty Acids in Human Neutrophils. Biomolecules. 2018; 8:144.

https://doi.org/10.3390/biom8040144

PMID:30428625

18. Legge $\mathrm{KL}$, Braciale TJ. Accelerated migration of respiratory dendritic cells to the regional lymph nodes is limited to the early phase of pulmonary infection. Immunity. 2003; 18:265-77. https://doi.org/10.1016/s1074-7613(03)00023-2 PMID: $\underline{12594953}$

19. Banchereau J, Steinman RM. Dendritic cells and the control of immunity. Nature. 1998; 392:245-52.

https://doi.org/10.1038/32588

PMID: $\underline{921319}$

20. Hashimoto S, Gon Y, Takeshita I, Maruoka S, Horie T. IL-4 and IL-13 induce myofibroblastic phenotype of human lung fibroblasts through c-Jun NH2-terminal kinase-dependent pathway. J Allergy Clin Immunol. 2001; 107:1001-8.

https://doi.org/10.1067/mai.2001.114702

PMID: 11398077

21. Parackova Z, Zentsova I, Bloomfield M, Vrabcova P, Smetanova J, Klocperk A, Mesežnikov G, Casas Mendez LF, Vymazal T, Sediva A. Disharmonic Inflammatory Signatures in COVID-19: Augmented Neutrophils' but Impaired Monocytes' and Dendritic Cells' Responsiveness. Cells. 2020; 9:2206.

https://doi.org/10.3390/cells9102206

PMID:33003471

22. Parackova Z, Vrabcova P, Zentsova I, Kayserova J, Richtrova I, Sojka L, Stechova K, Sumnik Z, Sediva A. Enhanced STAT3 phosphorylation and PD-L1 expression in myeloid dendritic cells indicate impaired IL-27Ralpha signaling in type 1 diabetes. Sci Rep. 2020; 10:493. https://doi.org/10.1038/s41598-020-57507-8 PMID:31949260

23. LeibundGut-Landmann $S$, Wüthrich $M$, Hohl TM. Immunity to fungi. Curr Opin Immunol. 2012; 24:449-58.

https://doi.org/10.1016/i.coi.2012.04.007 PMID:22613091

24. Romani L. Immunity to fungal infections. Nat Rev Immunol. 2011; 11:275-88.

https://doi.org/10.1038/nri2939

PMID:21394104

25. Roussey JA, Olszewski MA, Osterholzer JJ. Immunoregulation in Fungal Diseases. Microorganisms. 2016; 4:47. https://doi.org/10.3390/microorganisms4040047 PMID:27973396 
26. Fousert E, Toes R, Desai J. Neutrophil Extracellular Traps (NETs) Take the Central Stage in Driving Autoimmune Responses. Cells. 2020; 9:915.

https://doi.org/10.3390/cells9040915 PMID: 32276504

27. Stoiber W, Obermayer A, Steinbacher P, Krautgartner WD. The Role of Reactive Oxygen Species (ROS) in the Formation of Extracellular Traps (ETs) in Humans. Biomolecules. 2015; 5:702-23. https://doi.org/10.3390/biom5020702 PMID:25946076

28. Takesue $S$, Ohuchida $K$, Shinkawa $T$, Otsubo $Y$, Matsumoto S, Sagara A, Yonenaga A, Ando Y, Kibe S, Nakayama H, Iwamoto C, Shindo K, Moriyama T, et al. Neutrophil extracellular traps promote liver micrometastasis in pancreatic ductal adenocarcinoma via the activation of cancer-associated fibroblasts. Int J Oncol. 2020; 56:596-605.

https://doi.org/10.3892/ijo.2019.4951 PMID:31894273

29. Wu R, Duan L, Cui F, Cao J, Xiang Y, Tang Y, Zhou L. S100A9 promotes human hepatocellular carcinoma cell growth and invasion through RAGE-mediated ERK1/2 and p38 MAPK pathways. Exp Cell Res. 2015; 334:228-38.

https://doi.org/10.1016/i.yexcr.2015.04.008 PMID:25907296

30. Yue Q, Liu T, Cheng Z. Protective Effect of Colchicine on LPS-Induced Lung Injury in Rats via Inhibition of $\mathrm{P}$ 38, ERK1/2, and JNK Activation. Pharmacology. 2020; 105:639-44.

https://doi.org/10.1159/000504759

PMID:32688359

31. Condon TV, Sawyer RT, Fenton MJ, Riches DW. Lung dendritic cells at the innate-adaptive immune interface. J Leukoc Biol. 2011; 90:883-95.

https://doi.org/10.1189/jlb.0311134

PMID:21807741
32. Yang $D$, Chu $H$, Hou $Y$, Chai $Y$, Shuai $H$, Lee $A C$, Zhang $X$, Wang $Y$, Hu B, Huang $X$, Yuen TT, Cai JP, Zhou J, et al. Attenuated Interferon and Proinflammatory Response in SARS-CoV-2-Infected Human Dendritic Cells Is Associated With Viral Antagonism of STAT1 Phosphorylation. J Infect Dis. 2020; 222:734-45. https://doi.org/10.1093/infdis/iiaa356 PMID:32563187

33. Lippitsch A, Baal N, Chukovetskyi Y, Cunningham S, Michel G, Dietert K, Gurtner C, Gruber AD, Bein G, Hackstein H. Plasmacytoid dendritic cell depletion modifies FoxP3+ $\mathrm{T}$ cell homeostasis and the clinical course of bacterial pneumonia in mice. J Leukoc Biol. 2019; 106:977-85.

https://doi.org/10.1002/JLB.3AB0119-014RR

PMID:31265764

34. Chen X, Guo H, Qiu L, Zhang C, Deng Q, Leng Q. Immunomodulatory and Antiviral Activity of Metformin and Its Potential Implications in Treating Coronavirus Disease 2019 and Lung Injury. Front Immunol. 2020; 11:2056.

https://doi.org/10.3389/fimmu.2020.02056

PMID:32973814

35. Nath N, Khan M, Paintlia MK, Singh I, Hoda MN, Giri S. Metformin attenuated the autoimmune disease of the central nervous system in animal models of multiple sclerosis. J Immunol. 2009; 182:8005-14. https://doi.org/10.4049/jimmunol.0803563 PMID:19494326 


\section{SUPPLEMENTARY MATERIALS}

\section{Supplementary Figure}

A Control

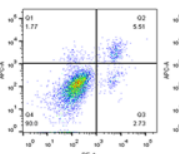

$\mu \mathrm{M}$

OA

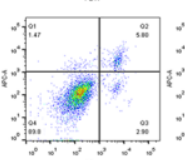

\begin{tabular}{ll|l|}
$00 \mu \mathrm{M}$ & & की \\
$\mathrm{OA}$ & & \\
\hline
\end{tabular}

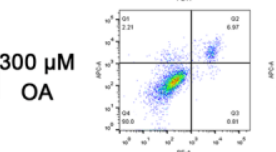

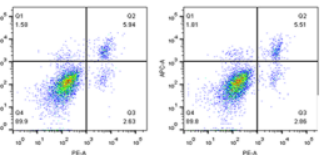
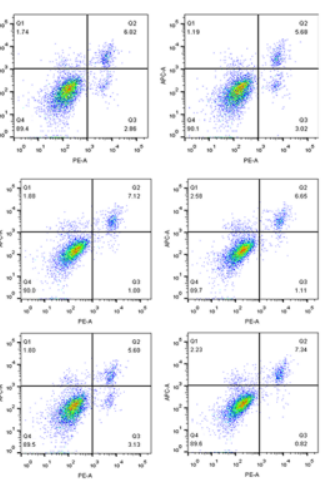

B

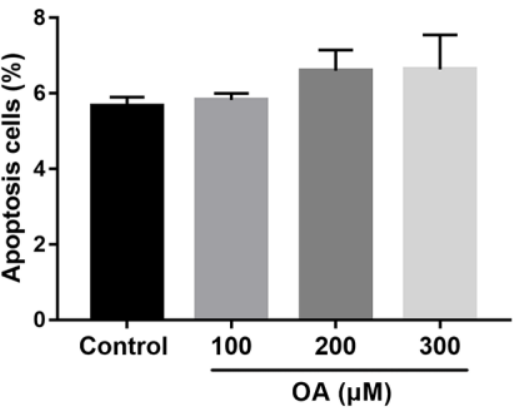

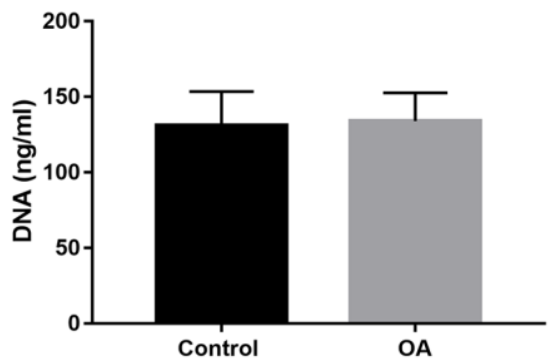

Supplementary Figure 1. The effects of OA on DCs. (A) Effects of OA on DCs apoptosis. (B) Effects of OA on DCs-DNA content. Abbreviations: OA: oleic acid; DCs: dendritic cells. 